\title{
TITLE:
}

\section{Weak and Strong Wave Turbulence Spectra for Elastic Thin Plate}

$\operatorname{AUTHOR}(S)$ :

Yokoyama, Naoto; Takaoka, Masanori

\section{CITATION:}

Yokoyama, Naoto ...[et al]. Weak and Strong Wave Turbulence Spectra for Elastic Thin Plate. Physical Review Letters 2013, 110(10): 105501.

ISSUE DATE:

2013-03

URL:

http://hdl.handle.net/2433/172224

RIGHT:

(C) 2013 American Physical Society 


\title{
Weak and Strong Wave Turbulence Spectra for Elastic Thin Plate
}

\author{
Naoto Yokoyama* \\ Department of Aeronautics and Astronautics, Kyoto University, Kyoto 606-8501, Japan \\ Masanori Takaoka ${ }^{\dagger}$ \\ Department of Mechanical Engineering, Doshisha University, Kyotanabe 610-0394, Japan
}

(Received 15 April 2012; revised manuscript received 3 December 2012; published 5 March 2013)

\begin{abstract}
A variety of statistically steady energy spectra in elastic wave turbulence have been reported in numerical simulations, experiments, and theoretical studies. Focusing on the energy levels of the system, we perform direct numerical simulations according to the Föppl-von Kármán equation, and successfully reproduce the variability of the energy spectra by changing the magnitude of external force systematically. When the total energies in wave fields are small, the energy spectra are close to a statistically steady solution of the kinetic equation in the weak turbulence theory. On the other hand, in large-energy wave fields, another self-similar spectrum is found. The coexistence of the weakly nonlinear spectrum in large wave numbers and the strongly nonlinear spectrum in small wave numbers is also found in moderate energy wave fields.
\end{abstract}

DOI: 10.1103/PhysRevLett.110.105501

PACS numbers: $62.30 .+\mathrm{d}, 05.45 .-\mathrm{a}, 46.40 .-\mathrm{f}$

There exist two types of "turbulence theories" started by Kolmogorov and Zakharov. The first, based on the selfsimilarity and the dimensional analysis, is applied on vortical flows governed by the Navier-Stokes equation. The second, based on the random phase approximation, is applied on wave fields, and is called the weak turbulence theory (WTT) [1]. While Kolmogorov's turbulence theory is applicable to strongly nonlinear turbulence, WTT, which is mathematically sophisticated, is applicable to weakly nonlinear wave fields [2,3].

Elastic waves propagating in thin elastic plates are considered a testing ground for numerical or experimental verification of the applicability of WTT. The kinetic equation obtained in WTT has two statistically steady solutions [4]. One is in a nonequilibrium state, where the azimuthally integrated energy spectrum $\mathcal{E}(k)$ as a function of wave numbers $k$ is given as $\mathcal{E}(k) \propto k\left[\log \left(k_{*} / k\right)\right]^{1 / 3}$. The other is in a thermal equilibrium state, where $\mathcal{E}(k) \propto k$. The function form of the nonequilibrium solution is determined so that the energy flux is constant, but the large cutoff wave number $k_{*}$ is indeterminate in the framework of WTT. Additionally, WTT cannot determine the direction of the energy flux in the nonequilibrium state in the elastic wave turbulence [1]. In these respects, the elastic wave turbulence is atypical as a weak turbulence system.

Düring et al. [4] obtained the energy spectrum corresponding to $\mathcal{E}(k) \propto k\left[\log \left(k_{*} / k\right)\right]^{1 / 3}$ by a direct numerical simulation (DNS). On the other hand, energy spectra close to $\mathcal{E}(k) \propto k^{-0.2}$ are reported in experiments using thin elastic steel plates $[5,6]$. The difference in the energy spectra is explained in terms of the anisotropy of the system in Ref. [7], and the discreteness of the numerical simulation in Ref. [8]. Moreover, dimensional analysis based on the self-similarity predicts energy spectra corresponding to the energy cascade, $\mathcal{E}(k) \propto k^{-1}$, and to the wave action cascade, $\mathcal{E}(k) \propto k^{-1 / 3}$ [9].

We optimistically believe that there should exist a simple unified explanation for the variability of the energy spectra including the theoretically predicted spectra. In this work, a series of DNS is performed according to a basic equation. Nonequilibrium steady wave turbulent states are obtained by adding external forces to small wave numbers and dissipation to large wave numbers. The numerical results provide a unified perspective on the variability of the spectra.

The governing equation for the lateral displacement $\zeta$ and the momentum $p$ in a thin elastic plate is called the Föppl-von Kármán (FvK) equation [10],

$$
\begin{aligned}
\partial_{t} p & =-\frac{E h^{2}}{12\left(1-\sigma^{2}\right)} \Delta^{2} \zeta+\{\zeta, \chi\}, \quad \partial_{t} \zeta=\frac{p}{\rho}, \\
\Delta^{2} \chi & =-\frac{E}{2}\{\zeta, \zeta\},
\end{aligned}
$$

where $\chi$ is the Airy stress potential. The Laplace operator and the Monge-Ampère operator are expressed as $\Delta$ and $\{f, g\}=\partial_{x x} f \partial_{y y} g+\partial_{y y} f \partial_{x x} g-2 \partial_{x y} f \partial_{x y} g$, respectively. The Young's modulus $E$, the Poisson ratio $\sigma$, and the density $\rho$ are the physical properties of the plate. The thickness of the plate is expressed by $h$. The FvK equation (1) holds even for large displacements when the gradient of the displacement is smaller than unity.

The linear dispersion relation between a wave number vector $\boldsymbol{k}$ and the corresponding frequency $\omega_{\boldsymbol{k}}$ is given as

$$
\omega_{k}=\sqrt{\frac{E h^{2}}{12\left(1-\sigma^{2}\right) \rho}} k^{2},
$$


where $k=|k|$. The complex amplitude $a_{k}=\left(\rho \omega_{k} \tilde{\zeta}_{k}+\right.$ $\left.i \tilde{p}_{k}\right) / \sqrt{2 \rho \omega_{k}}$ represents an elementary wave, where $\tilde{p}_{\boldsymbol{k}}$ and $\tilde{\zeta}_{k}$ are the Fourier components of $p$ and $\zeta$, respectively. The governing equation can be rewritten as

$$
\dot{a}_{k}=-i \omega_{k} a_{k}+\mathcal{N}_{k},
$$

where $\mathcal{N}_{\boldsymbol{k}}$ symbolically expresses the four-wave nonlinear interaction terms. Note that no three-wave interactions exist in this system. (See Ref. [4] for the nonlinear terms.) This equation can also be written in Hamiltonian form, $i \dot{a}_{k}=\delta \mathcal{H} / \delta a_{k}^{*}$, where $\delta / \delta a_{k}^{*}$ denotes the functional derivative with respect to the complex conjugate of $a_{k}$.

WTT can be applied to the elastic wave turbulence, if the nonlinear interactions among $a_{k}$ are weak and the random phase approximation is valid. The wave action $n_{k}$ is defined as $\left\langle a_{\boldsymbol{k}} a_{\boldsymbol{k}^{\prime}}^{*}\right\rangle=n_{\boldsymbol{k}} \delta_{\boldsymbol{k} \boldsymbol{k}^{\prime}}$, where $\langle\cdots\rangle$ expresses the ensemble average, and $\delta_{k k^{\prime}}$ is Kronecker's delta. When the system is statistically isotropic, we obtain the relation $\mathcal{E}(k) \propto k \omega_{k} n_{k}$. WTT considers the energy transfer due to the weakly nonlinear resonant interactions among four wave numbers. We have confirmed that this system has a huge number of the quartets which exactly satisfy the fourwave resonant conditions. Then, the system is little affected by the numerical discretization and it never goes into the frozen turbulence [11].

To investigate the statistically steady states, the following forcing and dissipative mechanisms are added to Eq. (3) as is usually done in turbulence simulations. Energy is input at small wave numbers and dissipated at large ones. In the forced wave numbers $|k| \leq 8 \pi$, the absolute value of the complex amplitude $\left|a_{k}\right|$ is kept constant as $C$ by multiplying the factor $C /\left|a_{k}\right|$ at each time step to control the energy of the system easily. In spite of fixing the absolute value, the phase of each mode evolves according to Eq. (3). The artificial eighth-order hyperviscosity $-\nu\left(|\boldsymbol{k}| / k_{d}\right)^{8} a_{\boldsymbol{k}}$ is added to the right-hand side of Eq. (3), where $\nu$ is a dissipation coefficient and $k_{d}$ is a dissipation wave number. The periodic boundary condition is adopted to match the assumptions in turbulence theory. The pseudospectral method with the aliasing removal by the $4 / 2$ law is employed to obtain the nonlinear terms. The fourth-order Runge-Kutta method is employed for the time integration, and the linear dispersive term and dissipative term are implicitly solved to improve numerical stability. Independently of the initial conditions, the system is attracted to a statistically steady state where the external force and the dissipation balance.

The material properties in the experiment with steel by Boudaoud et al. [5] are adopted in the present numerical simulations. Namely, $\rho=7.8 \times 10^{3} \mathrm{~kg} / \mathrm{m}^{3}$, $E=2.0 \times 10^{11} \mathrm{~Pa}, \sigma=0.30$, and $h=5.0 \times 10^{-4} \mathrm{~m}$, respectively. The plate is supposed to have the periodic boundary $1 \mathrm{~m} \times 1 \mathrm{~m}$.

A series of DNS with $512^{2}$ ( $256^{2}$ alias-free) modes [12] is performed in nine energy levels E1-E9. The energy levels are set by doubling the constant $C$ in the forcing term. The ratio of the stretching energy, which is the nonlinear part of the Hamiltonian, to the bending energy, which is the linear part of the Hamiltonian, represents the level of the nonlinearity that the entire wave field has. The ratios are on average $1 / 600$ in $E 1,1 / 20$ in $E 5$, and $1 / 4$ in $E 9$. Note that the FvK equation is justified since the root mean squares of the gradients of the displacements $\left\langle|\nabla \zeta|^{2}\right\rangle^{1 / 2}$ are, respectively, $5.0 \times 10^{-3}$ in $E 1,4.3 \times 10^{-2}$ in $E 5$, and $4.8 \times 10^{-1}$ in $E 9$. The standard deviations of the lateral displacements, $\left\langle\zeta^{2}\right\rangle^{1 / 2}$, are $1.9 \times 10^{-4} \mathrm{~m}$ in $E 1$, $1.4 \times 10^{-3} \mathrm{~m}$ in $E 5$, and $1.4 \times 10^{-2} \mathrm{~m}$ in $E 9$. The energy spectra for $E 1-E 9$ are drawn in Fig. 1. These spectra are time averaged during the statistically steady states, and the fluctuations are too small to affect the power-law exponents. Note that all the spectra in $|\boldsymbol{k}| \leq 8 \pi$ are proportional to $k^{3}$ owing to the forcing term.

We observe the spectral similarity in the low energy levels, i.e., in E1-E3. In the small energies, that is, when the nonlinearity is weak, the energy spectra approach the spectrum predicted by WTT, $\mathcal{E}(k) \propto k\left[\log \left(k_{*} / k\right)\right]^{1 / 3}$, which fits the spectra by choosing $k_{d}=144 \pi$ as $k_{*}$. As the energy levels get elevated, i.e., in E4-E6, the downward-sloping spectrum spreads from the range with smaller wave numbers. We observe another spectral similarity in the high energy levels, i.e., in $E 7-E 9$, where the nonlinearity is relatively strong. The energy spectra are estimated as $\mathcal{E}(k) \propto k^{-0.30 \pm 0.04}$ by the method of least squares. From now on, for simplicity we use the expression

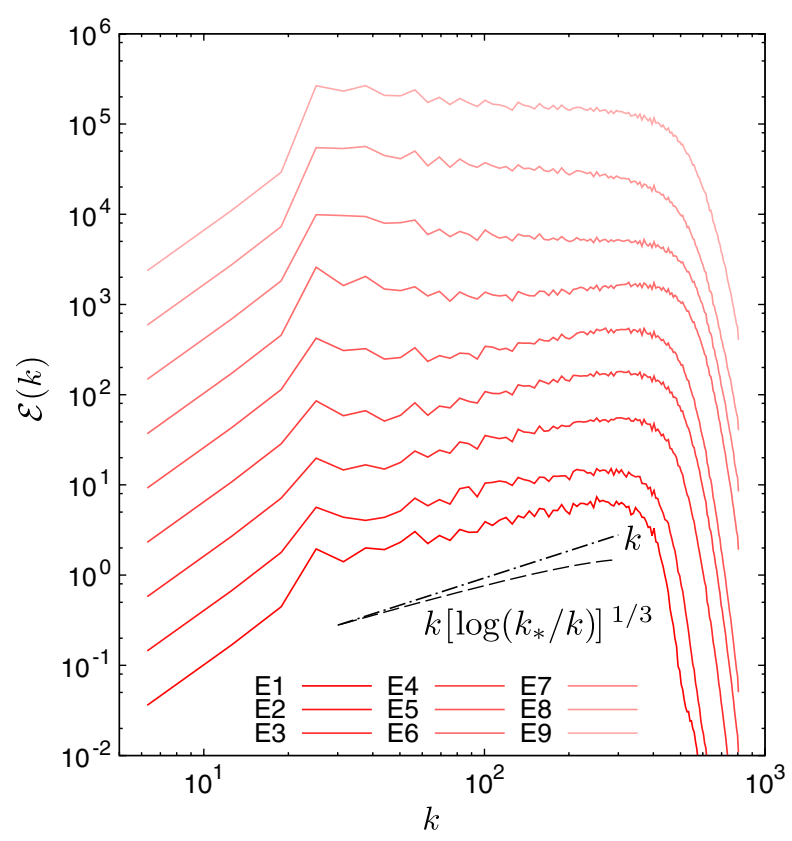

FIG. 1 (color online). Variability of the energy spectra due to energy levels. The energy levels are numbered from bottom to top. The dash-dotted line and the broken curve, respectively, show $k$ and $k\left[\log \left(k_{*} / k\right)\right]^{1 / 3}$, where $k_{*}=144 \pi$. 
"strongly nonlinear" for the relatively strongly nonlinear regime where WTT is not justified. For a wave field with the energy between $E 6$ and $E 7$, one may observe $\mathcal{E}(k) \propto$ $k^{-0.2}$ as in Refs. [5,6], if one forcibly fit the spectra to a single self-similar spectrum. Though their experimental energy spectra appear to collapse to a similar function form, the change of the energies is as small as one or two decades. In the experiment on decaying turbulence where the change of the energies is relatively large [7], the energy spectra approach the prediction of WTT as the energy decays. Our simulations reproduce all these experimental results by changing the magnitude of the external force as shown in Fig. 1. Note that this reproducibility supports the applicability of the FvK equation in all these energy levels in addition to the spectral similarities mentioned above.

To investigate the nonlinearities in each scale, the time evolutions of the magnitudes of elementary waves, $\left|a_{k}\right|$, which would remain constant in the absence of the nonlinear interactions, are shown in Fig. 2. We select $\boldsymbol{k}=$ $(16 \pi, 0),(32 \pi, 0),(64 \pi, 0)$, and $(128 \pi, 0)$ as the representatives in each scale. Each evolution is drawn for three periods determined by the linear dispersion, $3 T_{k}=$ $6 \pi / \omega_{k}$. As the energy becomes larger from $E 1$ to $E 9$, the fluctuations of $\left|a_{k}\right|$ 's become larger and faster as the overall trend. In Fig. 2(a), i.e., in the large energy E9, all $\left|a_{k}\right|$ 's are far from constant. The nonlinear interactions are active in all the scales. On the other hand, in Fig. 2(c), i.e., in the small energy $E 1$, all $\left|a_{k}\right|$ 's are almost constant in time. The nonlinearity at each wave number is uniformly

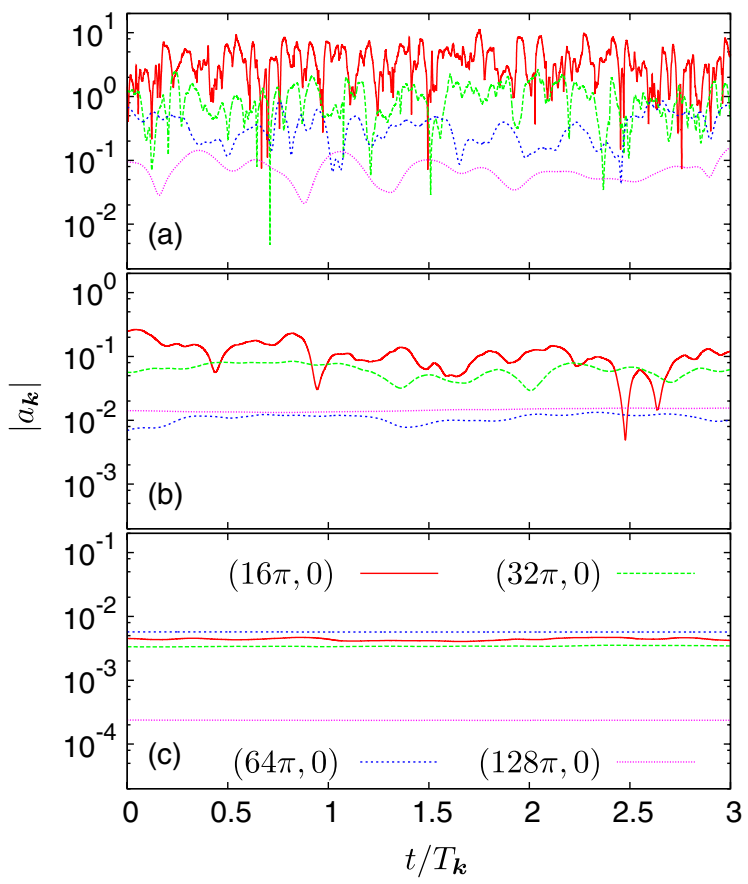

FIG. 2 (color online). The absolute values of the complex amplitudes $\left|a_{k}\right|$ during three periods. (a) Large energy E9, (b) moderate energy $E 5$, (c) small energy $E 1$. weak. Then, WTT is applicable for $E 1$, but not for $E 9$. In Fig. 2(b), i.e., in the moderate energy E5, the waves with smaller wave numbers have larger and faster fluctuations. It suggests that the failure of WTT, which appears at the wave numbers where the linear and nonlinear time scales are comparable [13], arises in the range with smaller wave numbers.

In Fig. 3, we examine the frequency spectrum $\left|\tilde{a}_{k}(\Omega)\right|^{2}$ of $a_{k}(t)$ at each wave number, where $\Omega$ is the angular frequency. The frequency spectra give the conjugate properties with the time evolution of $\left|a_{k}(t)\right|$ shown in Fig. 2. Note that if the nonlinear terms were neglected in Eq. (3), the frequency spectra would be the line segment at $\Omega / \omega_{k}=-1$. As shown in Figs. 3(a) and 3(b), for the higher energy levels and for the smaller wave numbers, the frequency spectra become broader, and the peaks of the frequency spectrum distribution shift more to the (negatively) larger frequencies from the linear dispersion relation [14]. For the strongly nonlinear waves, the linear dispersion relation does not characterize the system. When the energy is small, in Fig. 3(c), the peak frequencies almost coincide with those given by the linear dispersion relation. Even in this case the frequency shift to the larger frequencies in the small wave numbers is found in the inset. It is consistent with the observations in the experiments [15], though the boundary conditions may slightly affect the deviation as they described.

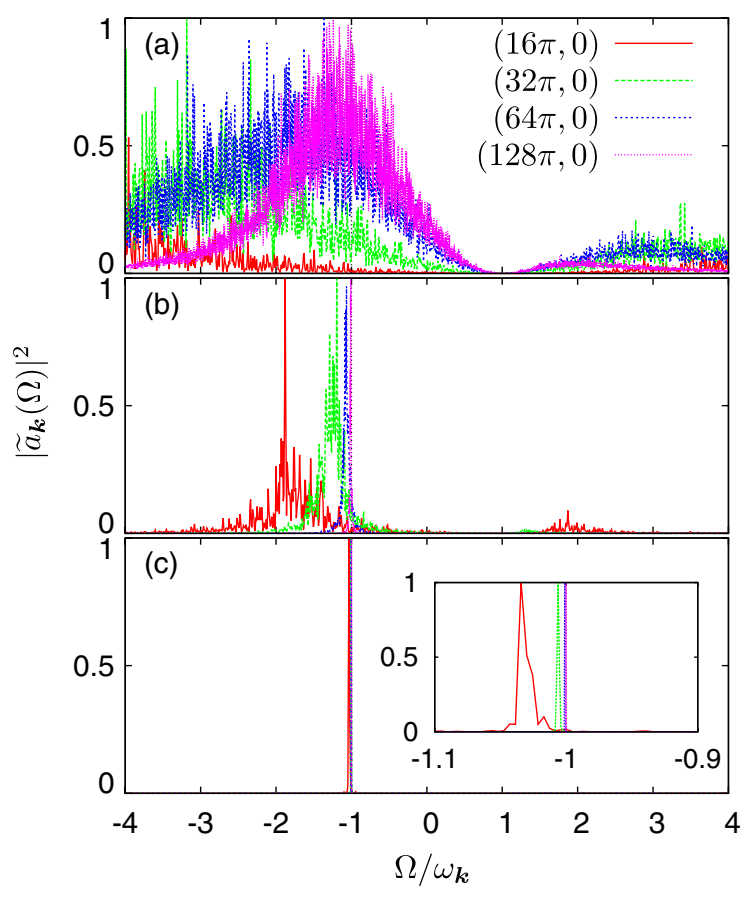

FIG. 3 (color online). The frequency spectrum $\left|\tilde{a}_{k}(\Omega)\right|^{2}$ at each wave number. (a) Large energy $E 9$, (b) moderate energy $E 5$, (c) small energy $E 1$, and the spectrum around $\Omega / \omega_{k}=-1$ is enlarged in the inset. Each spectrum is normalized for visibility so that its maximum is unity. 
The mean frequency shift is estimated by self-nonlinear interactions, which act as the dispersive term [3]. Then, Eq. (3) can be rewritten as $\dot{a}_{k}=-i\left(\omega_{k}+\Delta \omega_{k}\right) a_{k}+\mathcal{N}_{k}^{\prime}$, where $\mathcal{N}_{\boldsymbol{k}}^{\prime}$ expresses the nonself-nonlinear interactions. The mean frequency shift due to the self-nonlinear interactions is given as

$$
\Delta \omega_{k}=\sum_{\boldsymbol{k}^{\prime}} \frac{E}{4 \rho^{2} \omega_{\boldsymbol{k}} \omega_{\boldsymbol{k}^{\prime}}} \frac{\left|\boldsymbol{k} \times \boldsymbol{k}^{\prime}\right|^{4}}{\left|\boldsymbol{k}-\boldsymbol{k}^{\prime}\right|^{4}}\left(\left|a_{\boldsymbol{k}^{\prime}}\right|^{2}+\left|a_{-\boldsymbol{k}^{\prime}}\right|^{2}\right) .
$$

Suppose that the energy spectrum is self-similar as $\mathcal{E}(k) \propto$ $k^{\alpha}$ and therefore $\left|a_{k}\right|^{2} \propto k^{\alpha-3}$. Then, the summation converges if $-1<\alpha<3$, and the mean frequency shift is proportional to $k^{\alpha-1}$. For both the weak turbulence spectrum $(\alpha=1)$ and the strongly nonlinear spectrum ( $\alpha=-0.3$ shown in Fig. 1), the mean frequency shift is relatively large in the small wave numbers compared with the linear dispersion, $\omega_{k} \propto k^{2}$. The large mean frequency shift in the small wave numbers is consistent with the appearance of the strongly nonlinear spectra in the small wave numbers in Fig. 1.

We also examine the isotropy of the system by dividing the wave number plane azimuthally into eight regions. The maximal difference between the energy in one of the regions and the mean energy in the eight regions is used as a measure of isotropy. Even in the highest energy level $E 9$, the instantaneous relative difference is smaller than $10 \%$, and the time or ensemble averaging would make the difference smaller. Therefore, the wave field in $E 9$ is statistically isotropic. The isotropy is validated also by the two-dimensional energy spectrum. The isotropy in other energy levels is similarly confirmed. While the deviation of the power-law exponents observed in the earlier studies from the prediction of WTT is accounted for by the anisotropy in Ref. [7], the isotropy is maintained in all of our simulations. We therefore give an alternative mechanism for the deviation, which is accounted for by the nonlinearity.

In this Letter, focusing on energy levels, we have shown that the level of the nonlinearity provides the unified perspective on the variability of the spectra in the earlier studies [4-9]. The energy spectra in the low energy levels agree with the weak turbulence spectrum $\mathcal{E}(k) \propto$ $k\left[\log \left(k_{*} / k\right)\right]^{1 / 3}$. Less dissipative plates must be more weakly forced to reproduce the weak turbulence spectrum in experiments. The simulations also show another selfsimilar spectrum whose power-law exponent is approximately -0.30 in the high energy levels. The power-law exponent reminds us of $-1 / 3$ for the "inverse wave action cascade spectrum [16]," which follows from the dimensional analysis, in Ref. [9]. Note that the physical picture of the wave action is unclear in strongly nonlinear wave turbulence and the wave action is not conserved even under the kinetic equation in WTT of the present system owing to the $1 \rightarrow 3$ and $3 \rightarrow 1$ asymmetric resonant interactions.
Therefore, it is still an open question how the energy spectra in the high energy levels are created.

Moreover, in the moderate energy levels, the coexistence of the strongly nonlinear spectrum in the small wave numbers and the weakly nonlinear spectrum in the large wave numbers is found. The coexistence of the weakly and strongly nonlinear turbulence is predicted in several anisotropic wave turbulence systems [17]. In addition, the energy equipartition in the small wave numbers and the weak turbulence spectrum in the large wave numbers are simultaneously observed in a one-dimensional mathematical model of wave turbulence [18]. Since the FvK equation describes real physical dynamics, it is our future work to clarify the relation between real-space structures and the fluxes of conserved quantities.

This work was partially supported by the high technological research project of Doshisha University and MEXT, Japan. M. T. acknowledges partial support by KAKENHI Grant No. 22540402.

\footnotetext{
*yokoyama@kuaero.kyoto-u.ac.jp
}

†mtakaoka@mail.doshisha.ac.jp

[1] V.E. Zakharov, V.S. L'vov, and G. Falkovich, Kolmogorov Spectra of Turbulence I (Springer-Verlag, Berlin, 1992).

[2] V.E. Zakharov and N. N. Filonenko, Sov. Phys. Dokl. 11, 881 (1967); S. V. Nazarenko, A. C. Newell, and S. Galtier, Physica (Amsterdam) 152-153D, 646 (2001); Y. Lvov, S. Nazarenko, and R. West, Physica (Amsterdam) 184D, 333 (2003).

[3] S. Dyachenko, A.C. Newell, A. Pushkarev, and V.E. Zakharov, Physica (Amsterdam) 57D, 96 (1992).

[4] G. Düring, C. Josserand, and S. Rica, Phys. Rev. Lett. 97, 025503 (2006).

[5] A. Boudaoud, O. Cadot, B. Odille, and C. Touzé, Phys. Rev. Lett. 100, 234504 (2008).

[6] N. Mordant, Phys. Rev. Lett. 100, 234505 (2008).

[7] B. Miquel and N. Mordant, Phys. Rev. Lett. 107, 034501 (2011).

[8] B. Miquel and N. Mordant, Phys. Rev. E 84, 066607 (2011).

[9] S. Nazarenko, Wave Turbulence (Springer, Heidelberg, 2011).

[10] L. D. Landau and E. M. Lifshitz, Theory of Elasticity (Butterworth-Heinemann, Oxford, England, 1986); B. Audoly and Y. Pomeau, Elasticity and Geometry (Oxford University Press, Oxford, England, 2010).

[11] A. N. Pushkarev, Eur. J. Mech. B, Fluids 18, 345 (1999).

[12] It is not the memory but the CPU time that restricts the number of the modes because the time scale of the system is enormously wide as known from Eq. (2). Moreover, the time scale of the energy transfer due to the resonance interactions is extremely large in the weak turbulence.

[13] A. C. Newell, S. Nazarenko, and L. Biven, Physica (Amsterdam) 152-153D, 520 (2001). 
[14] The indistinct peak frequency of the mode $(16 \pi, 0)$ is found around $\Omega / \omega_{k} \approx-6$, which is out of the range of Fig. 3(a).

[15] P. Cobelli, P. Petitjeans, A. Maurel, V. Pagneux, and N. Mordant, Phys. Rev. Lett. 103, 204301 (2009).

[16] Though the cascade can be confirmed by constant flux, the flux stands little chance of being obtained in DNS of turbulence. The direction of the flux might be predicted by Fjørtoft argument, if the system had two positive conserved quantities to be cascaded.

[17] P. Goldreich and S. Sridhar, Astrophys. J. 438, 763 (1995); S. V. Nazarenko and A. A. Schekochihin, J. Fluid Mech. 677, 134 (2011); O. M. Phillips, J. Fluid Mech. 4, 426 (1958).

[18] D. Cai and D. W. McLaughlin, J. Math. Phys. (N.Y.) 41, 4125 (2000). 